\section{Estudo epidemiológico de base populacional sobre uso de medicamentos entre idosos na Região Metropolitana de Belo Horizonte, Minas Gerais, Brasil}

\author{
A population-based study on use of medication by \\ the elderly in Greater Metropolitan Belo Horizonte, \\ Minas Gerais, Brazil
}

\footnotetext{
${ }^{1}$ Núcleo de Estudos em Saúde Pública e Envelhecimento, Centro de Pesquisas René Rachou, Fundação Oswaldo Cruz/Universidade Federal de Minas Gerais, Belo Horizonte, Brasil. ${ }^{2}$ Faculdade de Medicina, Universidade Federal de Minas Gerais, Belo Horizonte, Brasil.

Correspondência A. I. Loyola Filho Núcleo de Estudos em Saúde Pública e Envelhecimento, Centro de Pesquisas René Rachou, Fundação Oswaldo Cruz/Universidade Federal de Minas Gerais. Av. Augusto de Lima 1715, Belo Horizonte, $M G$ 30190-002, Brasil. aloy@cpqrr.fiocruz.br
}

\begin{abstract}
The aim of this study was to investigate the use of medication and associated factors (sociodemographics, health conditions, and health services use) in a representative sample of 1,598 elderly individuals (60+ years) in Greater Metropolitan Belo Horizonte, Minas Gerais State, Brazil. The dependent variable was the number of drugs used in the preceding two weeks. Prevalence of medication was $72.1 \%$, and mean consumption was 2.18 drugs, most of which acting on the cardiovascular system. Use of medication (any amount) was independently associated with gender (female), age (80+ years), having consulted a physician, and presence of any chronic health condition. Use of five or more drugs was significantly associated with schooling ( $8+$ years, $O R=2.28)$, worse self-rated health (fair, $O R=5.45$; bad/very bad, $O R=5.35$ ). The results show that the types of medications used and factors associated with consumption were similar to those observed in other populations, suggesting some uniformity among various populations in the use of medication and its determinants.
\end{abstract}

Drug Utilization; Aged; Pharmacoepidemiology
Antônio I. de Loyola Filho 1,2

Elizabeth Uchoa 1,2

Maria Fernanda Lima-Costa 1,2

\section{Introdução}

A população idosa brasileira vem crescendo muito rapidamente a partir da segunda metade do século passado, tanto em números absolutos como relativos. Nos últimos quarenta anos, o número de habitantes com 60 ou mais anos de idade praticamente quintuplicou, saltando de 3 para 14 milhões 1 e, entre 1980 e 2000, a participação dos idosos na população total passou de $6,1 \%$ para $8,6 \%$ (Informações de saúde. http://www.datasus.gov.br, acessado em 15/Out/2004).

O envelhecimento populacional tem implicações sobre os serviços de saúde, em termos de capacidade de atendimento da demanda e de custeio. A maior convivência com problemas crônicos de saúde faz dos idosos grandes consumidores de serviços de saúde 2 e de medicamentos 3 . Nos países desenvolvidos, o uso de medicamentos entre idosos tem aumentado ao longo do tempo, assim como a parcela dos gastos com saúde debitados à assistência farma-cêutica, com o agravante de que, nessa faixa etária, os benefícios obtidos com a terapia medicamentosa hoje não significam uma redução futura no uso de medicamentos 4 .

No Brasil, o impacto do envelhecimento populacional sobre o setor saúde ganha dimensão e complexidade maiores. O Brasil ainda luta para superar problemas sanitários típicos de países menos desenvolvidos, como des- 
nutrição, dengue e malária, aos quais vêm juntar-se as doenças crônico-degenerativas comuns na velhice 5 . O enfrentamento de tal desafio pelos planejadores e administradores em saúde pública se dá num contexto de escassez crônica de recursos para o financiamento da atenção à saúde. No campo da assistência farmacêutica, o acesso da população ao medicamento é limitado e desigual, penalizando os indivíduos mais vulneráveis e de baixa renda. No setor público, os problemas de acesso ao medicamento têm origem na desarticulação entre os diversos níveis de gerenciamento da assistência farmacêutica, nos entraves no armazenamento e distribuição dos fármacos, que levam a uma baixa disponibilidade e descontinuidade da oferta ${ }^{6}$. Além disso, os preços dos medicamentos têm aumentado acima da inflação 7 . $\mathrm{O}$ gasto médio com medicamentos chega a comprometer aproximadamente um quarto da renda mensal média de mais da metade da população idosa brasileira 8 .

Para o idoso, os riscos envolvidos no consumo de medicamentos são maiores, se comparados aos do restante da população. Alterações na farmacodinâmica e farmacocinética dos medicamentos, decorrentes do processo de envelhecimento, tornam esse contingente populacional mais vulnerável a interações medicamentosas, efeitos colaterais e reações medicamentosas adversas ${ }^{3}$. Outros fatores que contribuem para a elevação do risco são o descumprimento do regime terapêutico (déficits cognitivos e funcionais dificultam o reconhecimento e memorização dos medicamentos), a automedicação e o uso indevido, por parte do profissional, do arsenal terapêutico disponível (prescrição de regimes terapêuticos complexos, polifarmacoterapia nem sempre justificável, ausência de correspondência entre diagnóstico e ação farmacológica) 3,9.

Estudos de base populacional mostram que, nos países desenvolvidos, idosos do sexo feminino e mais velhos 10,11,12,13, viúvos 13 e com pior situação sócio-econômica 12 consomem mais medicamentos. A utilização de medicamentos é maior também entre idosos que avaliam sua saúde como ruim ou muito ruim 11,13,14,15, que experimentam problemas crônicos de saúde 16,17 e que utilizam serviços de saúde 11,14. No geral, os grupos de medicamentos mais utilizados são aqueles de atuação sobre os sistemas cardiovascular e nervoso central 10,11,12,13.

No Brasil, pouco se conhece sobre a farmacoepidemiologia dos idosos, pois estudos de base populacional sobre o tema ainda são escassos. Miralles \& Kimberlin 7 investigaram o consumo de medicamentos entre idosos resi- dentes em três bairros da cidade do Rio de Janeiro, diferenciados quanto ao nível sócio-econômico. As características associadas ao uso de medicamentos diferiram entre os bairros investigados, variando também em função de ser o medicamento prescrito ou não. Outros dois estudos brasileiros foram desenvolvidos na cidade de Fortaleza, uma metrópole situada na região nordeste, 18 e em Bambuí, pequena cidade situada na Região Sudeste 19. Neles, foram investigados a prevalência e os fatores associados ao consumo de medicamentos. Sexo, idade, renda, estado de saúde e uso de serviços de saúde apresentaram associações independentes com o uso de medicamentos prescritos em ambos os estudos 18,19. Para os medicamentos não prescritos, no entanto, os resultados variaram: em Fortaleza, a automedicação esteve associada à renda e à autonomia 18 , ao passo que em Bambuí, sexo, consulta médica e consulta ao farmacêutico foram as variáveis associadas a essa prática 19 .

O presente trabalho foi desenvolvido na Região Metropolitana de Belo Horizonte, situada no Sudeste do Brasil, e refere-se ao estudo da prevalência e dos fatores associados ao consumo de medicamentos entre idosos residentes na comunidade. O estudo analisa o padrão de consumo de medicamentos pelos idosos, com os objetivos de: (a) estimar a prevalência e o consumo total de medicamentos; (b) identificar os medicamentos mais consumidos; e (c) investigar as características sócio-demográficas, as condições de saúde e a utilização de serviços de saúde associadas ao uso de medicamentos.

\section{Material e métodos}

\section{Área}

A Região Metropolitana de Belo Horizonte, situada no Estado de Minas Gerais, é composta por 24 municípios, sendo a terceira do país em tamanho da população $(4,4$ milhões de habitantes em 2000) e produção econômica. As tendências demográficas observadas na Região Metropolitana de Belo Horizonte são semelhantes àquelas verificadas para o restante do país, com um rápido e progressivo envelhecimento da sua população 20 .

\section{População estudada}

Este estudo é parte de um grande inquérito de saúde, conduzido na Região Metropolitana de Belo Horizonte entre maio e julho de 2003. A amostra para este inquérito foi delineada de 
forma a produzir estimativas para a população adulta residente nos 24 municípios que compõem a região metropolitana. A definição do tamanho da amostra (2.500 domicílios) baseouse na experiência de pesquisas semelhantes, desenvolvidas em outras regiões metropolitanas brasileiras, e considerou a possibilidade de perdas da ordem de $20 \%$ 20,21. Trata-se de uma amostra por conglomerados em dois estágios (unidade geográfica e domicílio, respectivamente). Os setores censitários do Instituto Brasileiro de Geografia e Estatística (IBGE) 21 foram utilizados como unidades primárias de seleção e o domicílio foi a unidade amostral. Todos os residentes nesses domicílios com 20 ou mais anos de idade foram selecionados para este inquérito, que incluiu 13.701 participantes (taxa de resposta $=79 \%$ ). Os participantes eram semelhantes à população total da Região Metropolitana de Belo Horizonte em relação à distribuição etária e ao sexo. Para o presente trabalho foram selecionados todos os 1.777 participantes deste inquérito com 60 ou mais anos de idade. Os dados foram coletados no domicílio do entrevistado, por meio de um questionário suplementar à Pesquisa de Emprego e Desemprego da Região Metropolitana de Belo Horizonte 20 . A equipe de coletores foi devidamente treinada pela Fundação João Pinheiro, órgão do governo do Estado de Minas Gerais e responsável pela pesquisa. Maiores detalhes podem ser vistos em outras publicações ${ }^{20,21}$.

\section{Variável dependente}

A variável dependente do estudo foi o número de medicamentos consumidos nas duas últimas semanas. Foram pesquisados: nome, número de dias em que usou o medicamento no período considerado, forma farmacêutica, dosagem e o fabricante do medicamento. Ao entrevistado foi solicitado mostrar a receita e a embalagem do medicamento, para conferência. O nome do medicamento, sua forma farmacêutica, a dosagem e o nome do fabricante foram utilizados na sua identificação. Após a identificação, os medicamentos foram desdobrados em seus princípios ativos e classificados de acordo com o Anatomical Therapeutic Chemical Index (ATC/DDD Index), desenvolvido pelo World Health Organization Collaborating Centre for Drug Statistics Methodology 22. Nessa classificação, são considerados o órgão ou sistema sobre o qual o medicamento atua, além de suas propriedades terapêuticas e farmacológicas. Para o presente trabalho, utilizouse as classificações dos medicamentos relativas aos níveis 1 (anatômico) e 2 (terapêutico).

\section{Variáveis independentes}

Três conjuntos de variáveis independentes foram considerados neste trabalho: características sócio-demográficas, condições de saúde e uso de serviços de saúde. As variáveis sócio-demográficas incluíram: sexo, idade, anos completos de escolaridade, situação conjugal, renda pessoal mensal (em salários mínimos nacionais vigentes à época da entrevista) e morar sozinho. Os indicadores da condição de saúde considerados foram auto-avaliação da saúde e diagnóstico médico anterior para algumas doenças ou condições crônicas selecionadas. A auto-avaliação da saúde foi determinada por meio da pergunta "De uma maneira geral, você considera sua saúde: muito boa, boa, razoável, ruim ou muito ruim?". A pergunta "Algum médico ou profissional de saúde já disse que você tinha..." foi utilizada para investigar a história de diagnóstico médico. Artrite/reumatismo, câncer, hipertensão, asma/bronquite, diabetes, angina, infarto, outra doença do coração, derrame, doença renal crônica, doença da coluna ou das costas e depressão foram as doenças ou condições crônicas consideradas. Com relação ao uso de serviços de saúde, utilizou-se o número de visitas médicas e de internações hospitalares nos últimos 12 meses.

As informações foram obtidas por meio de entrevistas domiciliares. No estudo, quando o participante não pôde responder ao questionário, outro morador o fez $(21,3 \%$ das entrevistas foram respondidas por outro morador), exceto para variáveis que requeriam julgamento pessoal, como é o caso da auto-avaliação da saúde. Nesse caso, foi criada uma categoria "nãoinformada" para evitar dados faltantes na matriz de regressão 21 .

\section{Análise dos dados}

A prevalência do consumo de medicamentos foi estimada pela proporção de entrevistados que relataram o uso de pelo menos um medicamento nas duas últimas semanas. Dado que o consumo total de medicamentos (número de medicamentos) não apresentou uma distribuição normal, essa variável foi categorizada em quartis, preservando a ausência de consumo como uma categoria em separado (categoria de referência). Essa estratificação gerou quatro níveis de consumo: nenhum (0 medicamento), baixo (1-2 medicamentos), intermediário (3-4 medicamentos) e elevado (5+ medicamentos). A análise bivariada foi baseada no teste do quiquadrado de Pearson. A análise multivariada 
foi baseada em odds ratios, estimados pelo modelo de regressão logística multinomial ${ }^{23}$. Nesta análise, as categorias relativas ao consumo total de medicamentos $(1-2 ; 3-4 ; 5+)$ foram comparadas à categoria de referência. Foram incluídas no modelo logístico inicial todas as variáveis que, na análise bivariada, apresentaram associação com uso de medicamentos em nível de significância inferior a 0,20. O nível de significância de 0,05 foi o critério adotado para permanência da variável no modelo logístico final. O programa Stata, versão 7.0, (Stata Corporation, College Station, Estados Unidos) foi usado na análise dos dados, tendo sido adotados os procedimentos desse programa para inquéritos populacionais com desenhos amostrais complexos (comando svy), levando em conta o peso do indivíduo na amostra.

Esta investigação foi aprovada pelo Comitê de Ética da Fundação Oswaldo Cruz (FIOCRUZ).

\section{Resultados}

Dos 1.777 idosos participantes do inquérito de saúde, 1.598 (89,9\%) participaram do presente trabalho; 179 não foram incluídos na análise porque não foi possível obter informações sobre o seu uso de medicamentos. Os idosos es-tudados apresentavam as seguintes características: $56,2 \%$ eram do sexo feminino, a média da idade era igual a 69,7 anos (mínima=60; máxima =97) e predominava a baixa escolaridade $(41,4 \%$ possuíam até quatro anos completos de escolaridade). As perdas $(\mathrm{n}=179)$ apresentaram características semelhantes à população estudada, no tocante às variáveis incluídas na investigação (dados não mostrados em Tabela).

A prevalência estimada do uso de medicamentos na população estudada foi igual a $72,1 \%$. $\mathrm{O}$ número médio de medicamentos consumidos foi igual a 2,18. A prevalência desse uso foi mais alta entre as mulheres, em comparação aos homens (79,3 vs 61,5\%), assim como a média do número de medicamentos consumidos (2,56 e 1,64, respectivamente). O consumo de medicamentos aumentou progressivamente com a idade, em ambos os sexos, alcançando o pico na faixa etária superior. O consumo elevado de medicamentos (5+) foi observado em 14,3\%; esta proporção foi igual a $10,5 \%$ entre os homens e $16,9 \%$ entre as mulheres (Tabela 1 ).

Na Tabela 2 estão descritos os grupos de medicamentos mais consumidos, de acordo com a classificação anatômica (Nível 1) e terapêutica (Nível 2) da ATC. Em relação à primeira, 52,0\% dos medicamentos consumidos atuam sobre o sistema cardiovascular, seguindo em ordem decrescente os medicamentos com ação sobre o sistema nervoso $(14,2 \%)$ e o trato alimentar e metabolismo (12,2\%). Considerando a classificação anátomo-terapêutica, entre os dez me-

Tabela 1

Distribuição proporcional (\%) do consumo de medicamentos e média do número de medicamentos utilizados entre idosos, segundo sexo e faixa etária. Região Metropolitana de Belo Horizonte, Minas Gerais, Brasil, 2003.

\begin{tabular}{|c|c|c|c|c|c|}
\hline Sexo/Faixa etária & Nenhum & $1-2$ & $3-4$ & $5+$ & Média (IC95\%) \\
\hline \multicolumn{6}{|l|}{ Homens (anos) } \\
\hline $60-69$ & 44,3 & 31,6 & 13,7 & 10,4 & $1,47(1,27-1,67)$ \\
\hline $70-79$ & 31,6 & 36,2 & 23,0 & 9,2 & $1,83(1,54-2,13)$ \\
\hline 80 ou + & 23,7 & 44,5 & 16,4 & 15,4 & $2,11(1,64-1,80)$ \\
\hline Todos & 38,5 & 34,3 & 16,7 & 10,5 & $1,64(1,48-1,80)$ \\
\hline \multicolumn{6}{|l|}{ Mulheres (anos) } \\
\hline $60-69$ & 23,8 & 36,8 & 26,1 & 13,3 & $2,29(2,09-2,49)$ \\
\hline $70-79$ & 17,9 & 32,7 & 29,2 & 20,3 & $2,79(2,49-3,09)$ \\
\hline 80 ou + & 15,7 & 32,9 & 28,8 & 22,6 & $3,01(2,53-3,50)$ \\
\hline Todos & 20,7 & 34,9 & 27,5 & 16,9 & $2,56(2,39-2,72)$ \\
\hline \multicolumn{6}{|c|}{ Homens e mulheres (anos) } \\
\hline $60-69$ & 32,8 & 34,5 & 20,7 & 12,0 & $1,93(1,79-2,08)$ \\
\hline $70-79$ & 23,1 & 34,0 & 26,8 & 16,0 & $2,43(2,20-2,65)$ \\
\hline $80 \mathrm{ou}+$ & 18,3 & 36,7 & 24,7 & 20,3 & $2,72(2,35-3,09)$ \\
\hline Todos & 27,9 & 34,7 & 23,1 & 14,3 & $2,18(2,07-2,30)$ \\
\hline
\end{tabular}


Distribuição dos medicamentos utilizados por idosos, segundo a classificação anátomo-terapêutica (níveis 1 e 2 da Anatomical Therapeutical Chemical Index - ATC). Região Metropolitana de Belo Horizonte, Minas Gerais, Brasil, 2003 *.

\begin{tabular}{|c|c|c|c|}
\hline Medicamentos & Código ATC & n & $\%$ \\
\hline Sistema cardiovascular & $\mathrm{C}$ & 1.739 & 52,0 \\
\hline Diuréticos & $\mathrm{CO3}$ & 491 & 14,7 \\
\hline Agentes sobre o sistema renina-angiotensina & C09 & 421 & 12,6 \\
\hline Bloqueadores dos canais de cálcio & $\mathrm{C08}$ & 243 & 7,3 \\
\hline Betabloqueadores & $\mathrm{CO}$ & 242 & 7,2 \\
\hline Cardioterápicos & C01 & 173 & 5,2 \\
\hline Antilipêmicos & $\mathrm{C} 10$ & 81 & 2,4 \\
\hline Outros & $\mathrm{CO2}-\mathrm{C} 04-\mathrm{CO}$ & 88 & 2,6 \\
\hline Sistema nervoso & N & 475 & 14,2 \\
\hline Psicoanalépticos & N06 & 141 & 4,2 \\
\hline Psicolépticos & N05 & 131 & 3,9 \\
\hline Antiepiléticos & N03 & 81 & 2,4 \\
\hline Analgésicos & N02 & 62 & 1,9 \\
\hline Outros & N01-N4-N07 & 60 & 1,8 \\
\hline Trato alimentar e metabolismo & A & 408 & 12,2 \\
\hline Medicamentos usados no diabetes & A10 & 208 & 6,2 \\
\hline Antiácidos/Antiulcerosos/Antiflatulentos & A02 & 81 & 2,4 \\
\hline Vitaminas & A11 & 62 & 1,9 \\
\hline Outros & $\star *$ & 57 & 1,7 \\
\hline Sangue e órgãos formadores de sangue & B & 246 & 7,4 \\
\hline Antiagregrantes & B01 & 225 & 6,7 \\
\hline Antianêmicos & B03 & 21 & 0,6 \\
\hline Sistema músculo-esquelético & M & 129 & 3,9 \\
\hline Antiinflamatórios e Anti-reumáticos & M01 & 92 & 2,8 \\
\hline Outros & $\star \star \star \star ~$ & 37 & 1,1 \\
\hline Preparados hormonais sistêmicos, exceto hormônios sexuais & $\mathrm{H}$ & 87 & 2,6 \\
\hline Terápicos tireoidianos & $\mathrm{HO3}$ & 62 & 1,9 \\
\hline Outros & $\mathrm{H} 02$ & 25 & 0,7 \\
\hline Sistema respiratório & $\mathrm{R}$ & 85 & 2,5 \\
\hline Antiasmáticos & R03 & 53 & 1,6 \\
\hline Outros & R01-R05-R06 & 32 & 1,0 \\
\hline Órgãos sensoriais & S & 49 & 1,5 \\
\hline Oftalmológicos & S01 & 43 & 1,3 \\
\hline Outros & S02-S03 & 6 & 0,2 \\
\hline Sistema genitourinário e hormônios sexuais & G & 46 & 1,4 \\
\hline Hormônios sexuais e moduladores sistema genital & G03 & 36 & 1,1 \\
\hline Urológicos & G04 & 10 & 0,3 \\
\hline Antiinfectivos de uso sistêmico & $\mathrm{J}$ & 45 & 1,4 \\
\hline Antibacterianos de uso sistêmico & J01 & 43 & 1,3 \\
\hline Outros & J02-J05 & 2 & 0,1 \\
\hline Outros medicamentos & D-L-P & 37 & 1,1 \\
\hline Total & & 3.345 & 100,0 \\
\hline
\end{tabular}

* Freqüências absolutas e relativas calculadas desconsiderando os medicamentos

para os quais não foi possível uma classificação $(n=161)$;

** A01-A03-A04-A05-A06-A07-A12-A13;

*** M02-M03-M04-M05. 
dicamentos mais consumidos figuram cinco com ação sobre o sistema cardiovascular: diuréticos $(14,7 \%)$, inibidores do sistema reninaangiotensina (12,6\%), beta-bloqueadores e bloqueadores dos canais de cálcio (14,5\% somados) e cardioterápicos (5,2\%). Seguem-se a estes, os antiagregantes plaquetários $(6,7 \%)$, medicamentos utilizados na diabetes $(6,2 \%)$, piscoanalépticos e psicolépticos ( $8,1 \%$ somados) e antiinflamatórios/anti-reumáticos (2,8\%).

Na Tabela 3 estão apresentados os resultados da análise bivariada da associação entre o número de medicamentos consumidos e as covariáveis consideradas neste estudo. Observese que todas as variáveis consideradas apresentaram, nessa análise, associações estatisticamente significantes $(\mathrm{p}<0,05)$ com o consumo de medicamentos, exceto morar sozinho ( $\mathrm{p}=$ 0,773).

Os resultados finais da análise multivariada das características sócio-econômicas, indicadores das condições de saúde e do uso de serviços de saúde associados ao consumo de medicamentos estão apresentados na Tabela 4 . Sexo (feminino), idade (80+ anos), auto-avaliação de saúde (razoável), número de condições crônicas (uma ou mais) e número de consultas médicas nos últimos 12 meses (três ou mais consultas) apresentaram associações positivas e independentes com o consumo de medicamentos nos três níveis considerados. Associações significantes com o consumo de 5 ou mais medicamentos foram encontradas para escolaridade mais alta (8+ anos) e pior percepção da saúde (ruim/muito ruim). Uma associação negativa e independente foi encontrada para consumo de 3-4 medicamentos e estado civil (viúvo).

\section{Discussão}

No presente trabalho, o consumo de medicamentos entre os idosos foi menor que o observado em países desenvolvidos 10,13,15, em termos da prevalência e do número médio de medicamentos utilizados. Em relação aos estudos brasileiros, a prevalência do uso de medicamentos foi também menor que a verificada na cidade de Bambuí 19, situada em Minas Gerais, e na cidade do Rio de Janeiro 7. O número médio de medicamentos consumidos observado neste trabalho foi menor que o observado entre idosos residentes no Rio de Janeiro 7 , mas maior que o observado entre idosos com pior nível sócio-econômico residentes na cidade de Fortaleza 18. Diferenças na prevalência e na intensidade do uso de medicamentos podem refletir diferenças entre as populações quanto ao estado de saúde, utilização de serviços e modelo de atenção à saúde, além de traços demográficos e culturais ligadas ao consumo de medicamentos, como sexo, idade e propensão ao uso ${ }^{10}$. O menor uso de medicamentos entre idosos residentes na Região Metropolitana de Belo Horizonte pode ser atribuído a um ou mais desses fatores. É preciso considerar ainda que a menor prevalência do uso de medicamentos na Região Metropolitana de Belo Horizonte, em comparação ao observado em Bambuí, pode ser conseqüente ao uso, em Bambuí, de um período recordatório maior (90 dias), em comparação ao usado neste estudo (15 dias). São necessários estudos mais abrangentes neste país para explicar diferenças no consumo de medicamentos entre populações diferentes.

Diversos trabalhos mostram que os medicamentos com ação sobre o aparelho cardiovascular são os mais consumidos por idosos 10,11, 12,19. Resultados semelhantes foram observados no presente trabalho. Com relação à classificação terapêutica, o predomínio de medicamentos anti-hipertensivos - diuréticos e medicamentos que atuam sobre o sistema reninaangiotensina responderam por cerca de um quarto do total de medicamentos utilizados - é coerente com o fato da hipertensão ser a condição crônica auto-referida mais freqüente entre idosos belo-horizontinos 20 .

Estudos recentes, conduzidos em diferentes países, têm mostrado que sexo (feminino) e idade (mais velha) são as características sóciodemográficas mais consistentemente associadas ao consumo de medicamentos 10,12,13,16,19,24, o que foi corroborado pelo nosso estudo. A explicação para associação positiva entre idade e o maior consumo de medicamentos reside na maior ocorrência de problemas de saúde nas idades mais avançadas, geralmente de longa duração e com maior grau de severidade, cujo tratamento e alívio de sintomas demanda terapia farmacológica 12,13. Com relação ao sexo, alguns estudos 25,26 mostraram que, na população geral, as diferenças de consumo de medicamentos por sexo podem ser debitadas essencialmente aos medicamentos usados na abordagem de condições de saúde inerentes ao sexo feminino, ligadas ao seu papel reprodutivo, como a gravidez e a contracepção. Todavia, essa explicação não se aplica aos idosos. Nessa faixa etária, problemas de saúde específicos do sexo feminino estão basicamente relacionados a déficits hormonais na menopausa, e nesse caso, não parece razoável supor que a maior utilização de medicamentos pelas mulheres possa ser de alguma forma atribuída aos medicamentos usados na terapia de reposição hor- 
Resultados da análise bivariada da associação entre o consumo de medicamentos, características sociodemográficas, indicadores da condição de saúde e do uso de serviços de saúde entre idosos. Região Metropolitana de Belo Horizonte, Minas Gerais, Brasil, 2003.

\begin{tabular}{|c|c|c|c|c|c|}
\hline Variáveis & Não usou (\%) & Usou 1-2 (\%) & Usou 3-4 (\%) & Usou 5+ (\%) & Valor de $\mathrm{p}^{\star}$ \\
\hline \multicolumn{6}{|l|}{ Sexo } \\
\hline Masculino & 56,0 & 40,2 & 29,4 & 29,9 & \\
\hline Feminino & 44,0 & 59,8 & 70,6 & 70,1 & $<0,001$ \\
\hline \multicolumn{6}{|l|}{ Idade (anos) } \\
\hline $60-69$ & 65,9 & 56,0 & 50,3 & 47,2 & \\
\hline $70-79$ & 25,8 & 30,6 & 36,2 & 35,0 & \\
\hline 80 ou + & 8,2 & 13,3 & 13,5 & 17,8 & $<0,001$ \\
\hline \multicolumn{6}{|l|}{ Estado conjugal** } \\
\hline Casado & 57,8 & 51,2 & 51,1 & 51,0 & \\
\hline Viúvo & 25,9 & 33,6 & 36,5 & 38,7 & \\
\hline Solteiro/Separado & 16,3 & 15,1 & 12,4 & 10,3 & 0,024 \\
\hline \multicolumn{6}{|c|}{ Escolaridade (anos completos) } \\
\hline $0-3$ & 37,0 & 40,5 & 47,0 & 43,4 & \\
\hline $4-7$ & 28,7 & 31,4 & 33,8 & 32,7 & \\
\hline 8 ou + & 34,4 & 28,2 & 19,2 & 23,9 & 0,003 \\
\hline \multicolumn{6}{|l|}{ Renda mensal } \\
\hline \multicolumn{6}{|l|}{ (salários mínimos) $)^{\star \star, \star \star \star}$} \\
\hline$<1$ & 33,9 & 37,3 & 47,8 & 43,6 & \\
\hline $1-2$ & 34,2 & 32,3 & 30,5 & 29,1 & \\
\hline$>2$ & 31,9 & 30,4 & 21,7 & 27,3 & 0,016 \\
\hline \multicolumn{6}{|l|}{ Morar sozinho } \\
\hline Não & 87,3 & 86,7 & 89,0 & 86,1 & \\
\hline Sim & 12,7 & 13,3 & 11,0 & 13,9 & 0,773 \\
\hline \multicolumn{6}{|c|}{ Auto-avaliação de saúde } \\
\hline Muito boa/Boa & 55,3 & 41,3 & 26,2 & 11,5 & \\
\hline Razoável & 14,5 & 29,4 & 44,2 & 46,2 & \\
\hline Ruim/Muito ruim & 2,0 & 8,3 & 13,7 & 20,6 & \\
\hline Não-informada\# & 28,3 & 21,0 & 15,9 & 21,8 & $<0,001$ \\
\hline \multicolumn{6}{|c|}{ Número de condições crônicas } \\
\hline \multicolumn{6}{|l|}{ diagnosticadas } \\
\hline Nenhuma & 65,2 & 20,9 & 7,9 & 3,5 & \\
\hline 1 & 20,5 & 42,7 & 24,1 & 12,6 & \\
\hline 2 & 10,8 & 22,5 & 27,8 & 25,6 & \\
\hline 3 ou + & 3,5 & 14,0 & 40,2 & 58,4 & $<0,001$ \\
\hline \multicolumn{6}{|c|}{ Hospitalização (últimos 12 meses) } \\
\hline Não & 93,6 & 90,2 & 81,1 & 66,9 & \\
\hline Sim & 6,4 & 9,8 & 18,9 & 33,1 & $<0,001$ \\
\hline \multicolumn{6}{|c|}{ Número de consultas médicas } \\
\hline \multicolumn{6}{|l|}{ (últimos 12 meses) } \\
\hline$<3$ & 74,0 & 44,8 & 27,5 & 9,6 & \\
\hline $3-5$ & 19,1 & 30,5 & 33,3 & 36,2 & \\
\hline 6 ou + & 6,8 & 24,7 & 39,3 & 54,2 & $<0,001$ \\
\hline
\end{tabular}

* Teste do qui-quadrado de Pearson; significativo quando <0,05;

** O cálculo dos porcentuais desconsiderou os indivíduos sem informação;

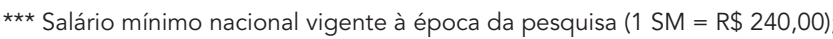

\# Outra pessoa (informante próximo) respondeu à pergunta. 
Resultado final da análise multivariada das características associadas ao consumo total de medicamentos. Região Metropolitana de Belo Horizonte, Minas Gerais, Brasil, 2003.

\begin{tabular}{|c|c|c|c|}
\hline Características & $\begin{array}{c}\text { 1-2 medicamentos } \\
\text { OR (IC95\%) }\end{array}$ & $\begin{array}{c}\text { 3-4 medicamentos } \\
\text { OR (IC95\%) }\end{array}$ & $\begin{array}{c}\text { 5+ medicamentos } \\
\text { OR (IC95\%) }\end{array}$ \\
\hline \multicolumn{4}{|l|}{ Sexo } \\
\hline Masculino & 1,00 & 1,00 & 1,00 \\
\hline Feminino & $1,84(1,27-2,66)$ & $2,98(1,91-4,66)$ & $2,82(1,64-4,85)$ \\
\hline \multicolumn{4}{|l|}{ Idade (anos) } \\
\hline $60-69$ & 1,00 & 1,00 & 1,00 \\
\hline $70-79$ & $1,32(0,90-1,95)$ & $1,71(1,07-2,74)$ & $1,56(0,91-2,69)$ \\
\hline 80 ou + & $2,40(1,33-4,33)$ & $3,17(1,60-6,29)$ & $3,95(1,77-8,82)$ \\
\hline \multicolumn{4}{|l|}{ Estado conjugal } \\
\hline Casado/Mora junto & 1,00 & 1,00 & 1,00 \\
\hline Viúvo & $0,78(0,50-1,21)$ & $0,53(0,32-0,90)$ & $0,59(0,32-1,12)$ \\
\hline Solteiro/Separado & $0,82(0,51-1,33)$ & $0,61(0,33-1,13)$ & $0,59(0,28-1,22)$ \\
\hline \multicolumn{4}{|c|}{ Escolaridade (anos completos } \\
\hline \multicolumn{4}{|l|}{ de freqüência à escola) } \\
\hline$<4$ & 1,00 & 1,00 & 1,00 \\
\hline $4-7$ & $1,31(0,89-1,94)$ & $1,30(0,83-2,04)$ & $1,36(0,80-2,31)$ \\
\hline $8 \mathrm{ou}+$ & $1,47(0,91-2,31)$ & $1,25(0,70-2,26)$ & $2,28(1,16-4,50)$ \\
\hline \multicolumn{4}{|c|}{ Auto-avaliação da saúde } \\
\hline Muito boa/Boa & 1,00 & 1,00 & 1,00 \\
\hline Razoável & $1,77(1,09-2,85)$ & $2,72(1,57-4,72)$ & $5,45(2,71-11,0)$ \\
\hline Ruim/Muito ruim & $2,05(0,83-5,10)$ & $2,30(0,85-6,19)$ & $5,35(1,80-15,88)$ \\
\hline Desconhecida & $0,90(0,61-1,33)$ & $0,86(0,51-1,44)$ & $2,38(1,19-4,76)$ \\
\hline \multicolumn{4}{|c|}{ Número de condições crônicas* } \\
\hline Nenhuma & 1,00 & 1,00 & 1,00 \\
\hline 1 & $6,12(4,16-9,02)$ & $8,42(4,59-15,47)$ & $11,58(3,91-34,29)$ \\
\hline 2 & $4,63(2,75-7,80)$ & $12,30(6,39-23,66)$ & $25,20(8,46-75,07)$ \\
\hline 3 ou + & $7,99(4,18-15,29)$ & $46,30(21,82-98,25)$ & $145,87(47,37-449,21)$ \\
\hline \multicolumn{4}{|c|}{ Número de consultas médicas } \\
\hline$<3$ & 1,00 & 1,00 & 1,00 \\
\hline $3-5$ & $2,17(1,44-3,28)$ & $2,91(1,78-4,78)$ & $8,08(4,14-15,77)$ \\
\hline 6 ou + & $3,91(2,35-6,51)$ & $6,88(3,95-11,98)$ & $23,25(11,67-46,33)$ \\
\hline
\end{tabular}

* Número de condições crônicas de saúde (ver seção metodológica).

Odds ratio (IC95\%) estimados pelo método de regressão logística multinomial.

Categoria referência $=$ indivíduos que não consumiram medicamentos.

Participaram da análise 1.566 indivíduos.

monal $(1,1 \%$ do total de medicamentos consumidos pelos idosos da Região Metropolitana de Belo Horizonte). O mais provável é que uma maior utilização de medicamentos pelas mulheres idosas possa estar ligada a outras questões, de ordem biológica (mulheres são mais afetadas por problemas de saúde não-fatais), psicológica (mulheres são mais conscientes dos sintomas físicos e colocam mais atenção sobre os seus problemas de saúde) e sócio-cultural (ao longo da vida, mulheres utilizam mais freqüentemente os serviços de saúde e estão mais familiarizadas com os medicamentos) 24 , questões essas, de alguma forma, potencializadoras do uso de medicamentos. Na população estudada, as mulheres apresentaram um maior número de doenças crônicas e visitaram mais vezes os médicos que os homens 20 , mas no pre- 
sente trabalho, a associação positiva entre sexo feminino e uso de medicamentos persistiu, mesmo após o ajustamento por essas variáveis. Dessa forma, o uso de serviços de saúde e o número de doenças crônicas explicam somente uma parte do maior uso de medicamentos entre as idosas.

Situação conjugal e escolaridade parecem ser menos importantes na determinação do consumo de medicamentos entre idosos. Vários estudos não encontraram associação entre essas variáveis e o consumo de medicamentos nessa faixa etária 7,11,14,17,19. Os resultados do presente trabalho reforçam essa observação, uma vez que somente associações pontuais foram observadas: o consumo de 3-4 medicamentos apresentou associação significante com viuvez e o consumo de 5 ou mais medicamentos apresentou associação com 8 ou mais anos de escolaridade.

O presente trabalho mostrou o uso elevado de medicamentos entre idosos com pior estado de saúde (pior auto-avaliação de saúde e maior número de condições crônicas), achados freqüentes em outros estudos farmacoepidemiológicos, tanto no Brasil 7,19 quanto em outros países 11,13,14,15,16. Da mesma forma, as associações positivas entre uso de serviços de saúde e consumo de medicamentos observadas na Região Metropolitana de Belo Horizonte foram também detectadas em outros estudos $11,17,19$. Esses achados são consistentes, pois uma conexão entre problemas de saúde, visitas médicas e uso de medicamentos é óbvia: as pessoas ficam (ou percebem-se) doentes, procuram os serviços de saúde em busca de solução ou alívio para os seus problemas e recebem dos médicos prescrições medicamentosas para tratá-los.

Diferentemente de outros países, no Brasil inexistem bancos de dados sobre consumo de medicamentos apropriados a pesquisas farmacoepidemiológicas abrangentes 27. Dessa forma, na presente investigação, como em outros estudos brasileiros 7,18,19, optou-se pela coleta de dados referentes ao uso de medicamentos por meio de entrevistas. A entrevista domiciliar é avaliada como a mais completa e confiável fonte de dados para a investigação do consumo de medicamentos entre idosos, em comparação a questionários postais e entrevistas por telefone 12. Além disso, como medicamentos prescritos e não-prescritos podem ser consumidos simultaneamente, optou-se por analisálos indistintamente. Embora isso tenha gerado dificuldades na comparação de nossos resultados com outros estudos que fizeram essa distinção na análise, nossa opção possibilitou captar um quadro explicativo mais próximo do consumo real de medicamentos pela população idosa 12 .

Nesta investigação, um pouco mais de um quinto das respostas $(21,3 \%)$ foi fornecida por informantes próximos, no caso, outro morador do domicílio 20. O consumo de medicamentos entre idosos com informante próximo foi menor que o observado entre idosos que responderam ao questionário. Além disso, os primeiros, em comparação aos últimos, apresentaram proporções significativamente maiores de homens, com idade igual ou superior a 80 anos e sem qualquer condição crônica presente. Em relação às demais variáveis associadas ao uso de medicamentos, não houve diferenciação entre eles. Como a exclusão do estudo daqueles idosos com informante próximo implicaria a perda de poder estatístico, sem produzir alterações substanciais no padrão de associações verificado, optou-se por considerá-los na análise. Para a variável auto-avaliação da saúde (de caráter subjetivo), os respondentes próximos constituíram uma categoria à parte (informação desconhecida).

Os resultados deste trabalho mostram que o tipo de medicamento consumido (em termos de classificação anátomo-terapêutica) e os fatores associados a esse consumo foram muito semelhantes ao observado em outras populações, sugerindo que existe uma certa uniformidade no consumo e nos seus determinantes, entre populações diferentes. As fortes associações encontradas entre consumo de medicamentos, condições de saúde e uso de serviços de saúde reforçam o reconhecimento da importância do medicamento na atenção à saúde do idoso. Esses achados, frente ao rápido envelhecimento populacional brasileiro e aos riscos potenciais do uso de medicamentos em idade mais avançada ${ }^{3}$, reforçam a necessidade de que a assistência farmacêutica ao idoso seja uma preocupação constante dos planejadores em saúde, no sentido de garantir a esse segmento populacional o acesso ao medicamento e o seu uso com qualidade. 


\section{Resumo}

Este estudo teve por objetivo investigar o consumo de medicamentos e fatores associados (sócio-demográficos, condições de saúde e uso de serviços de saúde) em uma amostra representativa de 1.598 pessoas com 60+ anos de idade, residentes na Região Metropolitana de Belo Horizonte, Minas Gerais, Brasil. A variável dependente foi o número de medicamentos consumido nas duas semanas precedentes. A prevalência do uso de medicamentos foi de $72,1 \%$ e a média de medicamentos consumidos igual a 2,18, predominando aqueles com ação sobre o sistema cardiovascular. O consumo de qualquer número de medicamentos apresentou associação independente com sexo feminino, idade (80+ anos), ter visitado um médico e apresentar alguma condição crônica. O consumo de maior número de medicamentos (5+) apresentou associações significativas com escolaridade $(8+$ anos, $O R=2,28)$ e pior auto-avaliação da saúde (razoável, $O R=5,45$; ruim/ muito ruim, $O R=5,35$ ). Os resultados deste trabalho mostram que o tipo de medicamento consumido e os fatores associados a esse consumo foram muito semelhantes ao observado em outras populações, sugerindo que existe uma certa uniformidade no consumo e nos seus determinantes, entre populações diferentes.

Uso de Medicamentos; Idoso; Farmacoepidemiologia

\section{Colaboradores}

A. I. Loyola Filho responsabilizou-se pela análise dos dados e redação do artigo. E. Uchoa colaborou na discussão dos resultados e revisou criticamente o texto final. M. F. Lima-Costa orientou o trabalho, supervisionou a redação e foi responsável por uma revisão crítica final do texto.

\section{Agradecimentos}

Agradecemos à Profa. Suely Rozenfeld, da Escola Nacional de Saúde Pública, Fundação Oswaldo Cruz, pelos valiosos esclarecimentos relativos ao uso do índice de classificação Anatomical Therapeutic Chemical Index.

\section{Referências}

1. Lima-Costa MF, Veras R. Saúde pública e envelhecimento [Editorial]. Cad Saúde Pública 2003; 19:700.

2. Desai MM, Zhang P, Hennessy CH. Surveillance for morbidity and mortality among older adults United States, 1995-1996. MMWR CDC Surveill Summ 1999; 48:7-25.

3. Tamblyn R. Medication use in seniors: challenges and solutions. Therapie 1996; 51:269-82.

4. O'Neill C, Hughes CM, Jamison J, Schweizer A. Cost of pharmacological care of the elderly. Drugs Aging 2003; 20:253-61.

5. Silva Jr. JB, Gomes FBC, Cezário AC, Moura L. Doenças e agravos não-transmissíveis: bases epidemiológicas. In: Rouquayrol MZ, Almeida-Filho $\mathrm{N}$, organizadores. Epidemiologia \& saúde. Rio de Janeiro: MEDSI Editora; 2003. p. 289-311.

6. Guerra Jr. AA, Acúrcio FA, Gomes CAP, Miralles M, Girardi SN, Werneck GAF, et al. Disponibilidade de medicamentos essenciais em duas regiões de Minas Gerais, Brasil. Rev Panam Salud Publica 2004; 15:168-75.

7. Miralles MA, Kimberlin CL. Perceived access to care and medication use among ambulatory elderly in Rio de Janeiro, Brazil. Soc Sci Med 1998; 46:345-55.

8. Lima-Costa MFF, Barreto SM, Giatti L. Condições de saúde, capacidade funcional, uso de serviços de saúde e gastos com medicamentos da população idosa brasileira: um estudo descritivo baseado na Pesquisa Nacional por Amostra de Domicílios. Cad Saúde Pública 2003; 19:735-43.

9. Rozenfeld S. Prevalência, fatores associados e mal uso de medicamentos entre idosos: uma revisão. Cad Saúde Pública 2003; 19:717-24.

10. Helling DK, Lemke JH, Semla TP, Wallace RB, Lipson DP, Cornoni-Huntley J. Medication use characteristics in the elderly: the Iowa 65+ Rural Health Study. J Am Geriatr Soc 1987; 35:4-12.

11. Chrischilles EA, Foley DJ, Wallace RB, Lemke JH, Semla TP, Hanlon JT, et al. Use of medications by persons 65 and over: data from the established populations for epidemiologic studies of the elderly. J Gerontol 1992; 47:M137-44.

12. Chen YF, Dewey ME, Avery AJ; The Analysis Group of the MRCCFA Study. Self-reported medication use for older people in England Wales. J Clin Pharm Thera 2001; 26:129-40.

13. Linjakumpu T, Hartikainen S, Klaukka T, Veijola J, Kivelä S-L, Isoaho R. Use of medications and polypharmacy are increasing among the elderly. J Clin Epidemiol 2002; 55:809-17.

14. Fillenbaum GG, Hanlon JT, Corder EH, ZiqubuPage T, Wall Jr. WE, Brock, D. Prescription and nonprescription drug use among black and White community-residing elderly. Am J Public Health 1993; 83:1577-82.

15. Rosholm JU, Christensen K. Relationship between drug use and self-reported health in elderly Danes. Eur J Clin Pharmacol 1997; 53:179-83.

16. Woo J, Ho SC, Yuen YK, Lau J. Drug use in an elderly Chinese population: prevalence and associated factors. Gerontology 1995; 41:98-108. 
17. Fillenbaum GG, Horner RD, Hanlon JT, Landerman LR, Dawson DV, Cohen HJ. Factors predicting change in prescription and nonprescription drug use in a community-residing black and white elderly population. J Clin Epidemiol 1996; 49:58793.

18. Coelho Filho KM, Marcopito LF, Castelo A. Perfil de utilização de medicamentos por idosos em área urbana do Nordeste do Brasil. Rev Saúde Pública 2004; 38:557-64.

19. Loyola Filho AI, Uchoa E, Firmo JOA, Lima-Costa MFF. Estudo de base populacional sobre o consumo de medicamentos entre idosos: Projeto Bambuí. Cad Saúde Pública 2005; 21:545-53.

20. Lima-Costa MFF. A saúde dos adultos na Região Metropolitana de Belo Horizonte: um estudo epidemiológico de base populacional. Belo Horizonte: Núcleo de Estudos em Saúde Pública e Envelhecimento, Fundação Oswaldo Cruz/Universidade Federal de Minas Gerais; 2004.

21. Fundação João Pinheiro. Pesquisa de Emprego e Desemprego na Região Metropolitana de Belo Horizonte (PED/RMBH): plano de trabalho. Belo Horizonte: Sistema Nacional de Emprego; 1997.
22. World Health Organization. Anatomical therapeutical chemical (ATC) classification index with defined daily doses (DDDs). http://www.whocc.no/ atcddd/indexdatabase/ (acessado em Jun/2004).

23. Long JS, Freese J. Regression models for categorical dependent variables using Stata. College Station: Stata Press; 2003.

24. Laukkanen P, Heikkinen E, Kauppinen M, Kallinen M. Use of drugs by non-institutionalized urban Finns born in 1904-1923 and the association of drug use with mood and self-rated health. Age Ageing 1992; 21:343-52.

25. Svarstad BA, Cleary PD, Mechanic D, Robers PA. Gender differences in the acquisition of prescribed drugs: an epidemiological study. Med Care 1987; 25:1089-98.

26. Zadoroznyj M, Svarstad BL. Gender, employment and medication use. Soc Sci Med 1990; 31:971-8.

27. Rozenfeld S, Valente J. Estudos de utilização de medicamentos - considerações técnicas sobre coleta e análise de dados. Epidemiol Serv Saúde 2004; 13:115-23.

Recebido em 05/Ago/2005

Versão final reapresentada em 16/Mar/2006 Aprovado em 20/Abr/2006 